\title{
Harvesting solar energy by flower-like carbon cloth nanocomposites for simultaneous generation of clean water and \\ electricity
}

You Xu, Zhenzhen Guo, Jun Wang, Zihe Chen, Jiacheng Yin, Zexian Zhang, Jiming Huang, Jingwen Qian and Xianbao Wang*

Hubei Collaborative Innovation Center for Advanced Organic Chemical Materials, Ministry-of-Education Key Laboratory for the Green Preparation and Application of Functional Materials, Hubei Key Laboratory of Polymer Materials (Hubei University), School of Materials Science and Engineering, Hubei University, Wuhan 430062, P.R. China;

Formula S1-4

Figure S1-23

Table S1-2

* Corresponding author. Tel.: +86-2788661729; Fax: +86-2788661729. 
E-mail: wangxb68@aliyun.com (X.B. Wang)

\section{Analysis of heat transfer processes}

Thermal analysis for the solar vapor desalination process is conducted, including the effective energy for evaporation $\left(Q_{\text {Evap }}\right)$, conductive heat loss to bulk water $\left(Q_{1}\right)$, convective $\left(Q_{2}\right)$ and radiative $\left(Q_{3}\right)$ heat loss to the surroundings.

The energy dynamic equilibrium can be expressed as:

$A \alpha q_{\text {Solar }}=Q_{\text {Evap }}+Q_{1}+Q_{2}+Q_{3}$

where $A$ denotes the surface area of absorber facing the sun, $\alpha$ solar absorbance, and $q_{\text {Solar }}$ input solar flux

The conductive heat loss to bulk water $\left(Q_{l}\right)$ can be calculated through the temperature gradient in the underlying water:

$Q_{1}=A k(T l 1-T l 2) / \Delta l$

where $k$ denotes thermal conductivity of bulk water. The temperature gradient in the underlying water below the samples is measured by two embedded thermocouples (i.e., $T l 1=27.2^{\circ} \mathrm{C}$ and $T l 2=26.8^{\circ} \mathrm{C}$ ). The distance between the thermocouples $\Delta l$ is $10 \mathrm{~mm}$. Consequently, the $Q_{2}$ is calculated to be $20.0 \mathrm{~J} \mathrm{~m}^{-2}$, corresponding $2.0 \%$.

The convective heat loss $\left(Q_{2}\right)$ to the adjacent environment can be calculated as:

$Q_{2}=A h\left(T_{a}-T_{\infty}\right)$

where $h$ is convection heat transfer coefficient (assumed to be $10 \mathrm{~W} \mathrm{~m}^{-2} \mathrm{~K}^{-1}$ ), $T_{a}$ is the top surface temperature of absorber $\left(35.3^{\circ} \mathrm{C}\right)$, and $T_{\infty}$ is the temperature of the adjacent environment. Since the light-absorbing material is surrounded by water layer and hot vapor, the adjacent temperature can be approximated as the vapor temperature (i.e., $T_{\infty}$ $=T_{\text {vapor }}=32.4{ }^{\circ} \mathrm{C}$ ). Consequently, the $Q_{3}$ is calculated to be $29.0 \mathrm{~J} \mathrm{~m}^{-2}$, corresponding $2.9 \%$.

The radiative heat loss $\left(Q_{3}\right)$ to the ambient environment can be calculated as: 
$Q_{3}=A \varepsilon \sigma\left(T_{a}^{4}-T_{\text {vapor }}^{4}\right)$

where $\varepsilon$ denotes the emission of the absorber (assumed to be 0.9531 ), and $\sigma$ is the Stefan-Boltzmann constant $\left(5.669 \times 10^{-8} \mathrm{~W} \mathrm{~m}^{-2} \mathrm{~K}^{-4}\right)$. Consequently, the $Q_{3}$ is calculated to be $10.7 \mathrm{~W} \mathrm{~m}^{-2}$, corresponding $1.1 \%$.
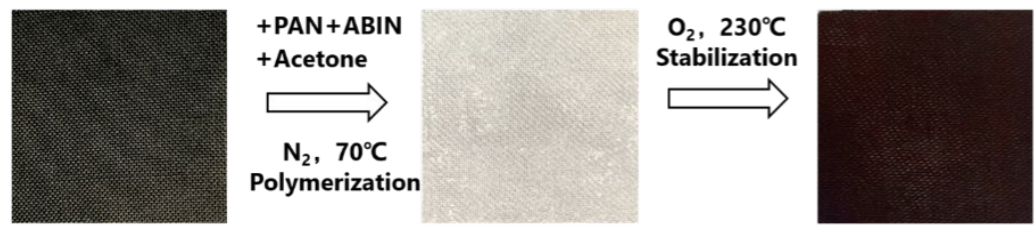

Figure S1. Optical images of the fabrication process of the CC-BPHPLS.

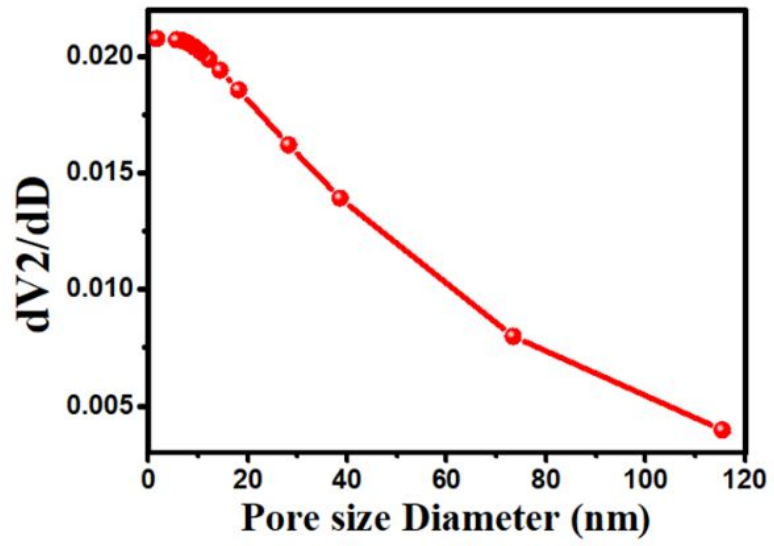

Figure S2. The differential pore size distribution of CC-BPHPLS.

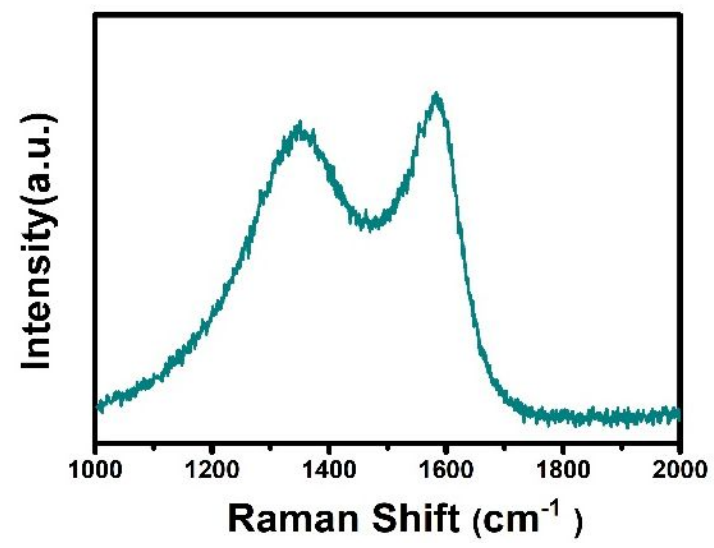

Figure S3. Raman spectra of CC-BPHPLS. 

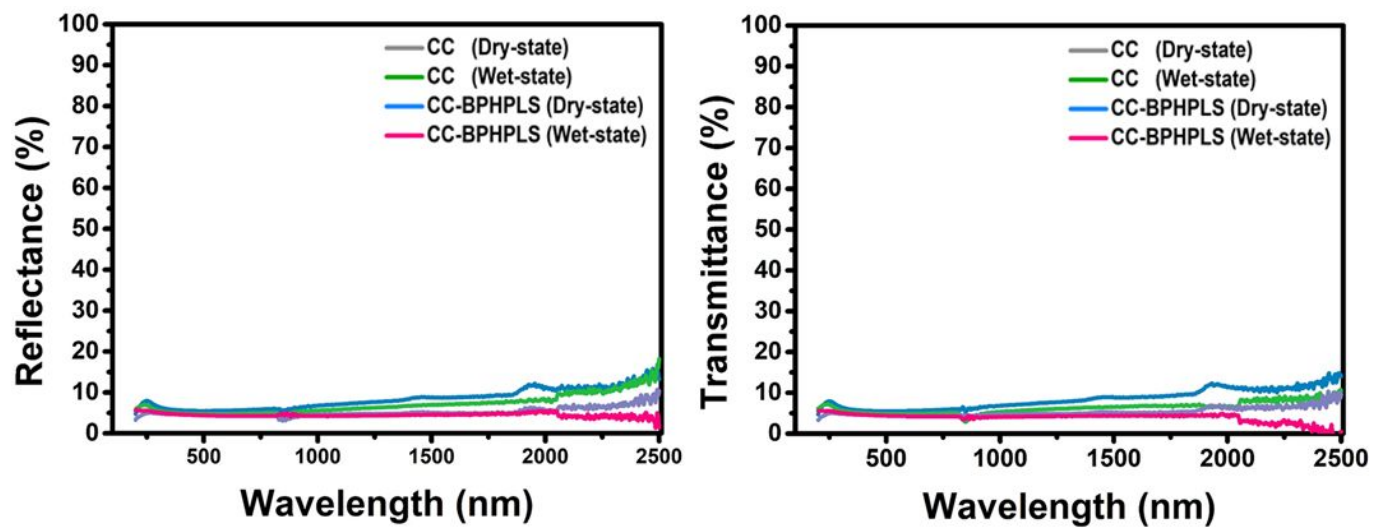

Figure S4. Reflectance and transmittance spectra of the wet/dry state CC and CC-BPHPLS in the wavelength range of 200-2500 $\mathrm{nm}$.
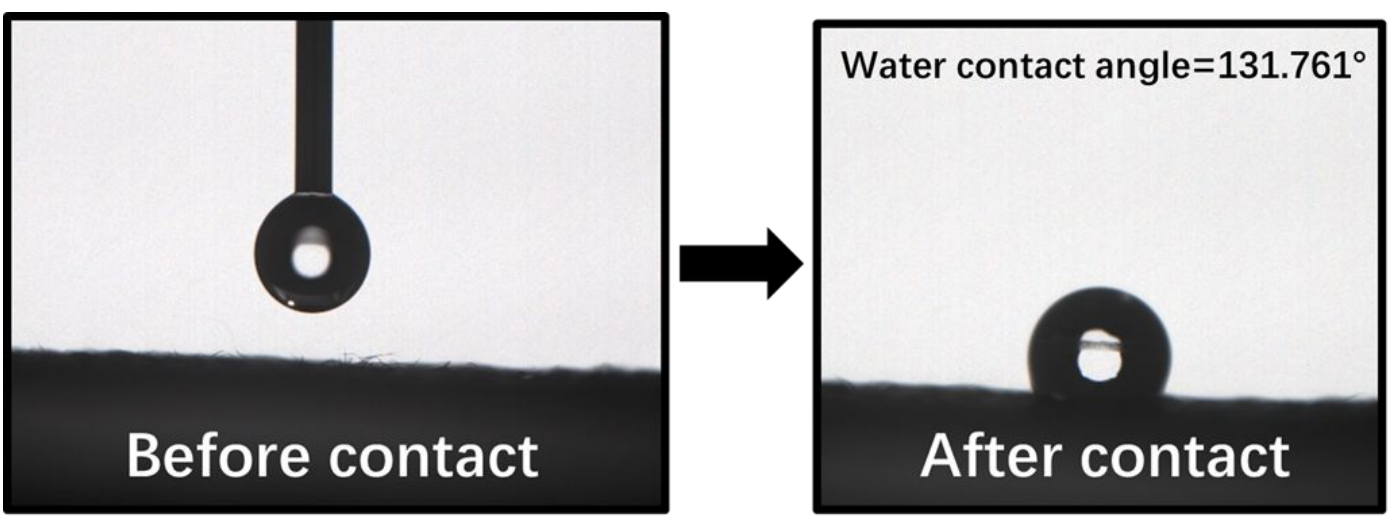

Figure S5. Water contact angles of the CC.

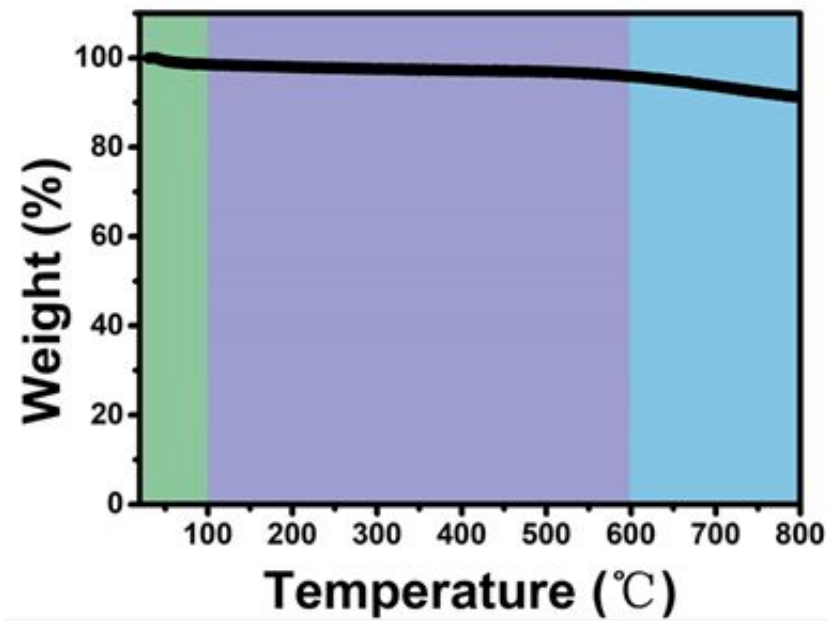

Figure S6. Thermogravimetric analysis (TGA) of the CC-BPHPLS from 30 to $800{ }^{\circ} \mathrm{C}$ in air atmosphere. 


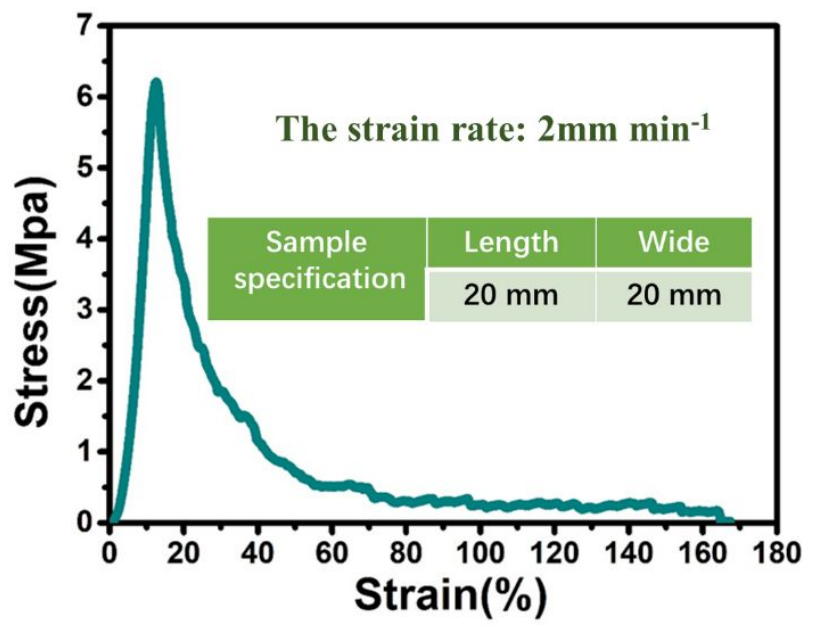

Figure S7. Stress-strain curves of the CC-BPHPLS.

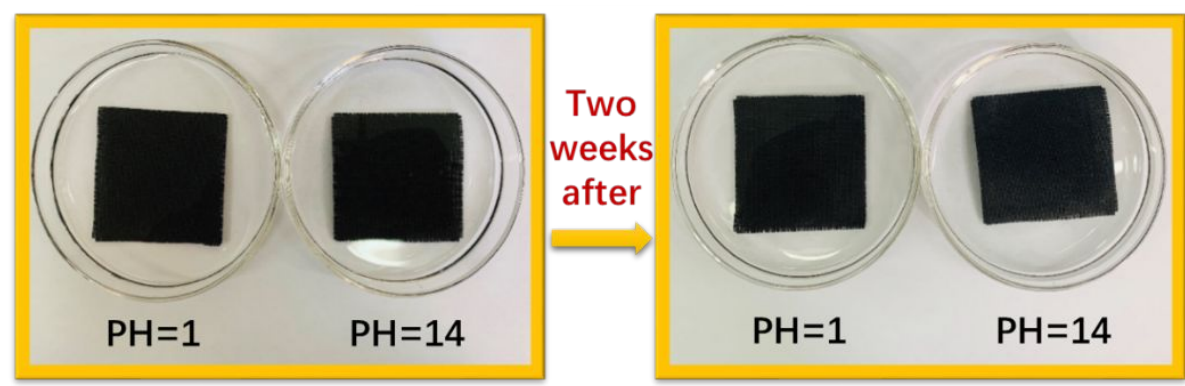

Figure S8. The state of the CC-BPHPLS immersed in acid and alkaline solution.

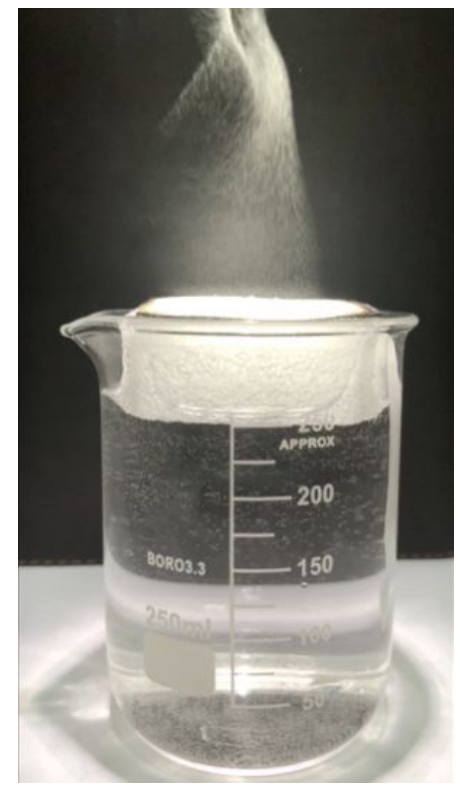

Figure S9. Solar steam generation performance of CC-BPHPLS under solar irradiance. 


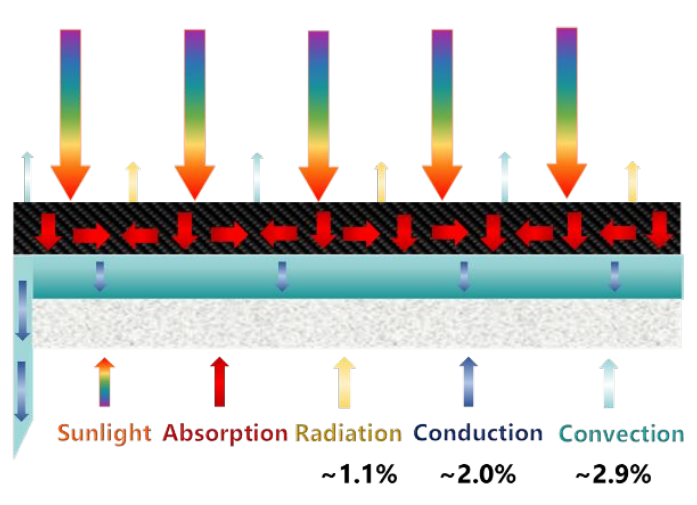

Figure S10. Energy balance and heat loss diagram of CC-BPHPLS during the solar vapor generation process.

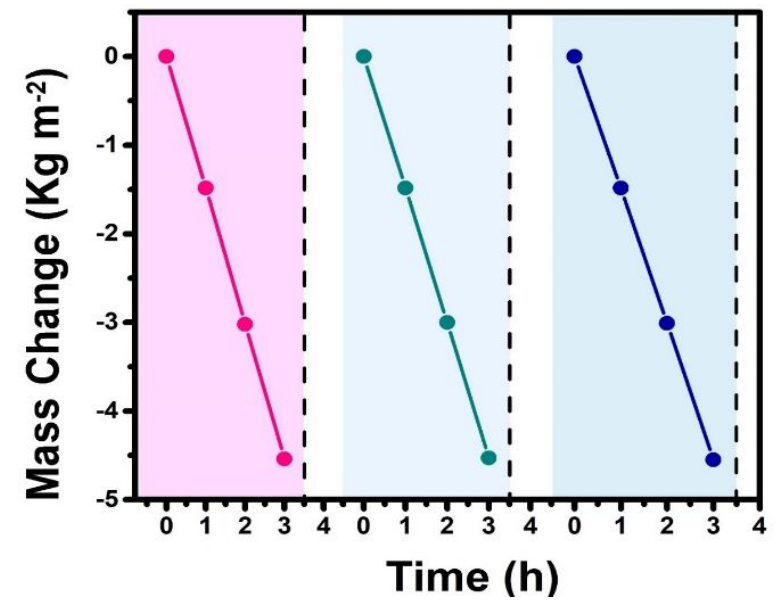

Figure S11. The durability test was performed under 1 sun illumination for $3 \mathrm{~h}$ a day.

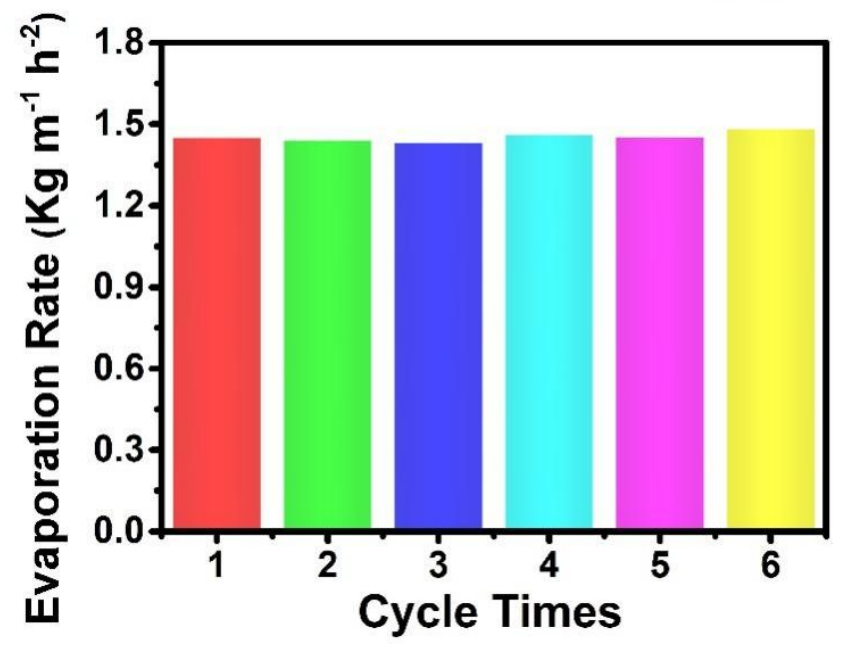


Figure S12. The desalination cycling test was performed under 1 sun illumination for $3 \mathrm{~h}$ a day.

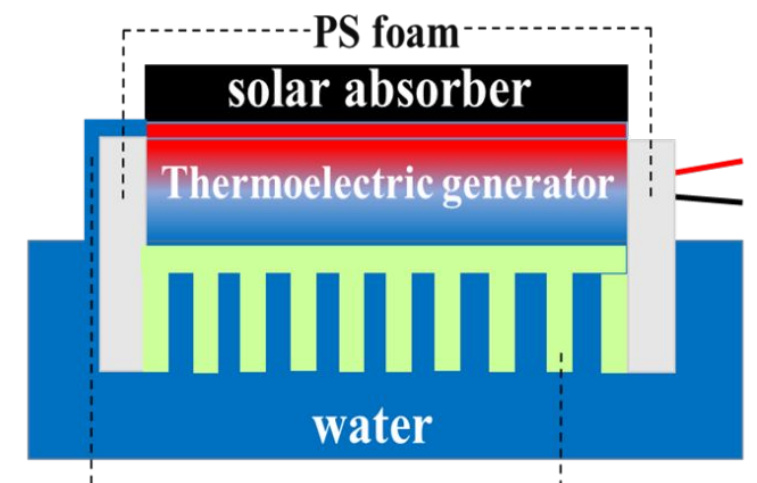

\section{Water transmission channel Radiating fin}

Figure S13. Schematic of the evaporation structure with TE and module water transmission channel.

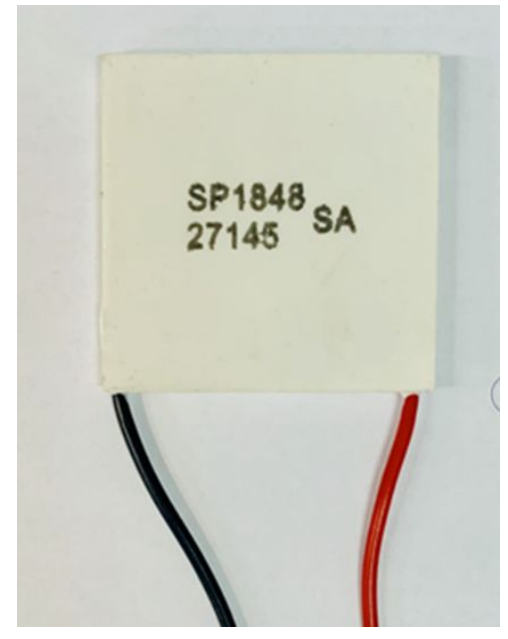

Figure S14. Photographs of the TE module.

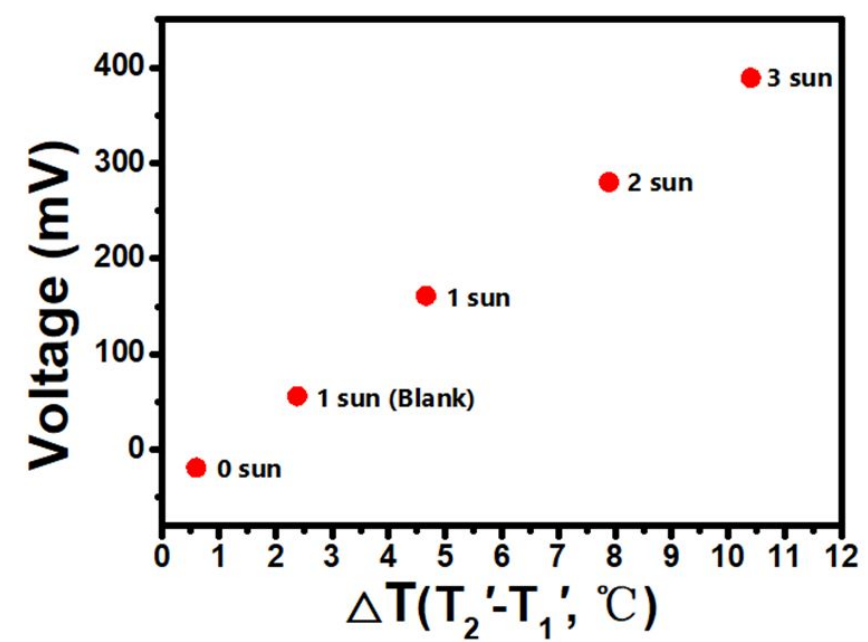

Figure S15. The temperature difference and open circuit voltage of TE module with water 
transmission channel at the steady-state water temperature platform of $25^{\circ} \mathrm{C}$.

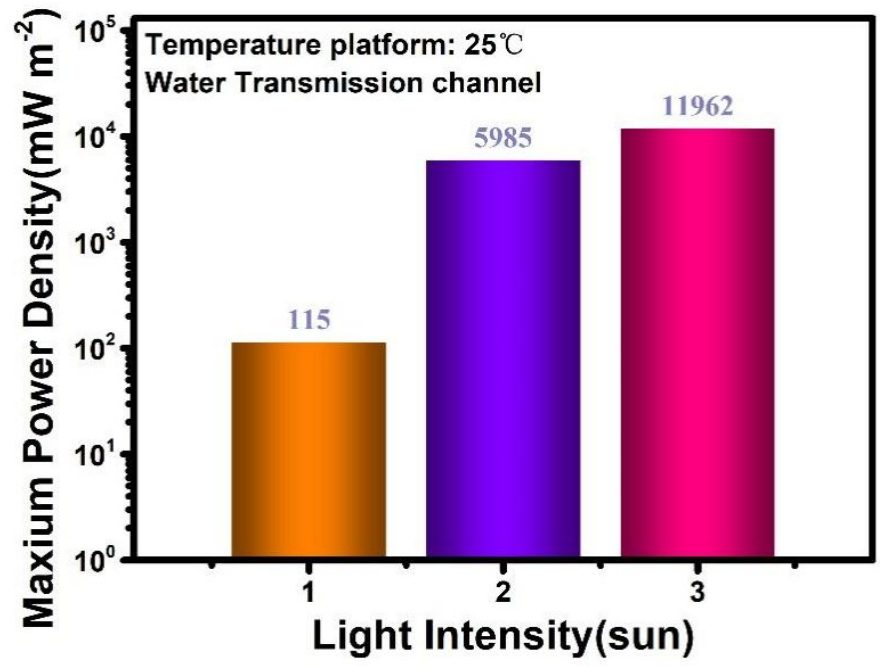

Figure S16. The maximum output power of the hybrid system under different light intensity.

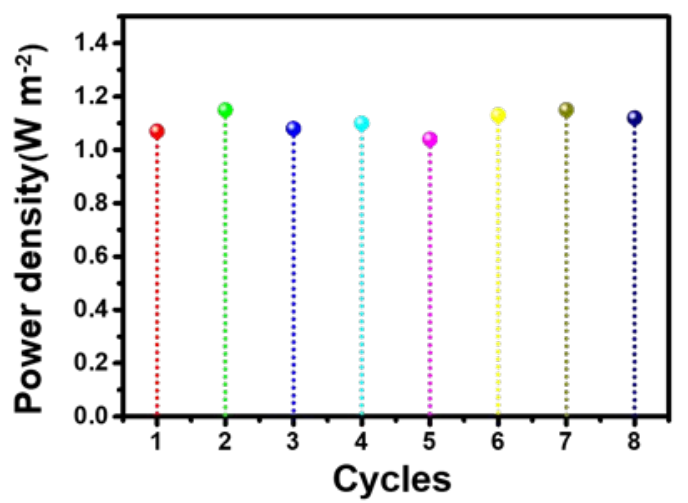

Figure S17. The cycling performance of hybrid system was performed at temperature platform of $25^{\circ} \mathrm{C}$ under 1 sun illumination.

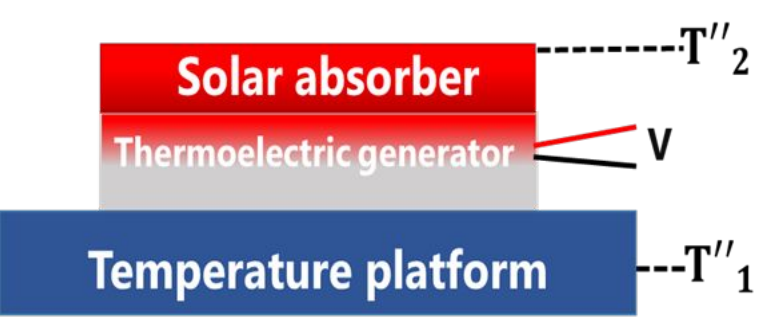

Figure S18. Schematic of thermoelectric power generation without water transmission channel under temperature platform. 

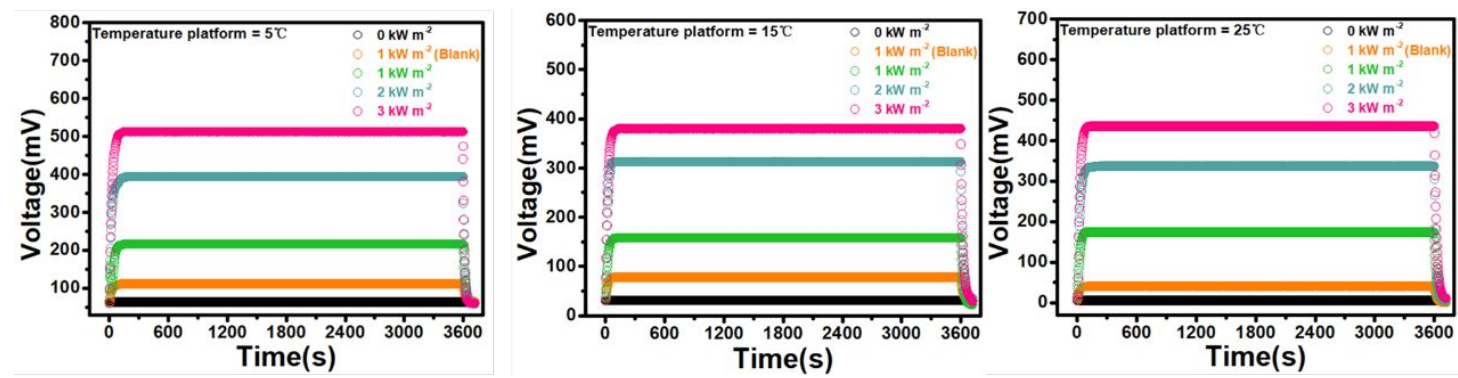

Figure S19. Light response of the TE device without water transmission channel at temperature platform of 5,15 , and $25^{\circ} \mathrm{C}$ under different solar irradiations.
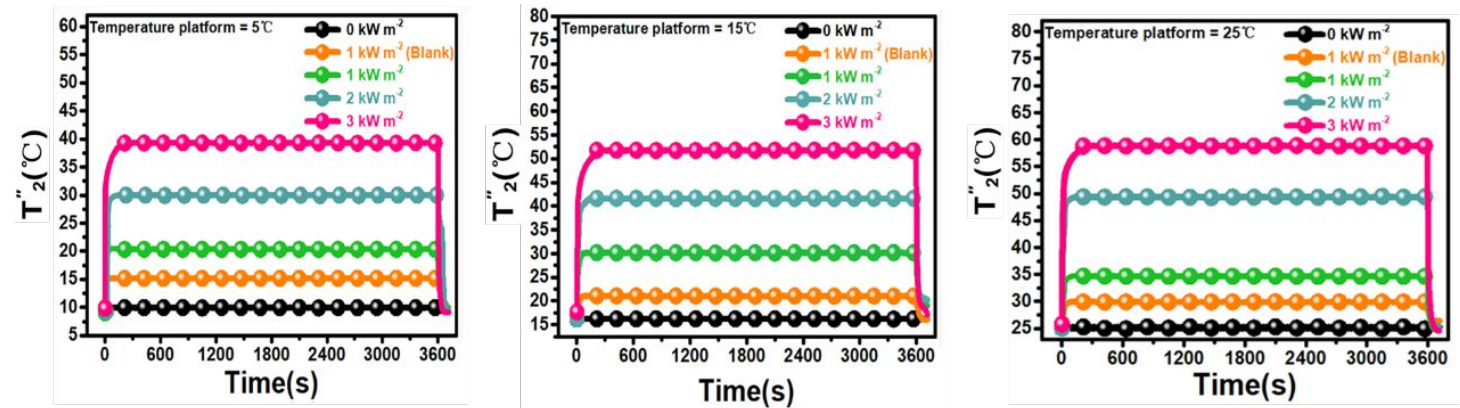

Figure S20. Temperature response of the TE device without water transmission channel at temperature platform of 5,15 , and $25{ }^{\circ} \mathrm{C}$ under different solar irradiations.
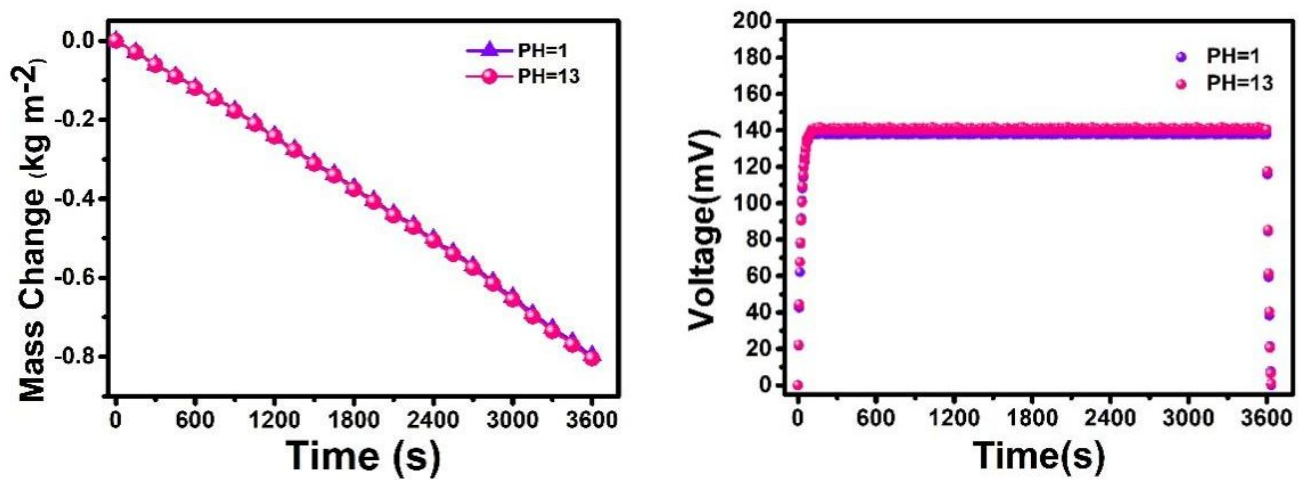

Figure S21. After submerging in a solution of $\mathrm{pH} 1$ and 13 for two weeks, time-dependent mass change of CC-BPHPLS with TE module (left) and the open circuit voltages of device with water transmission channel at temperature platform of $25^{\circ} \mathrm{C}$ under optical densities of $1 \mathrm{~kW} \mathrm{~m}^{-2}$ (right). 


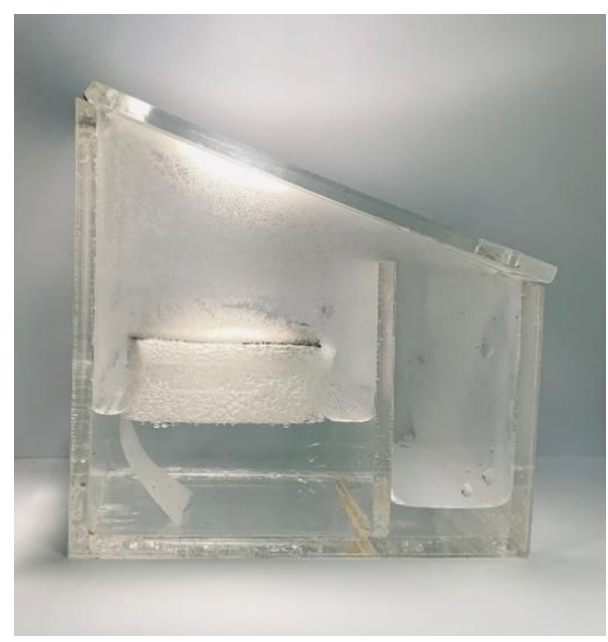

Figure S22. Schematic illustration of fresh water production based on the CC-BPHPLS.

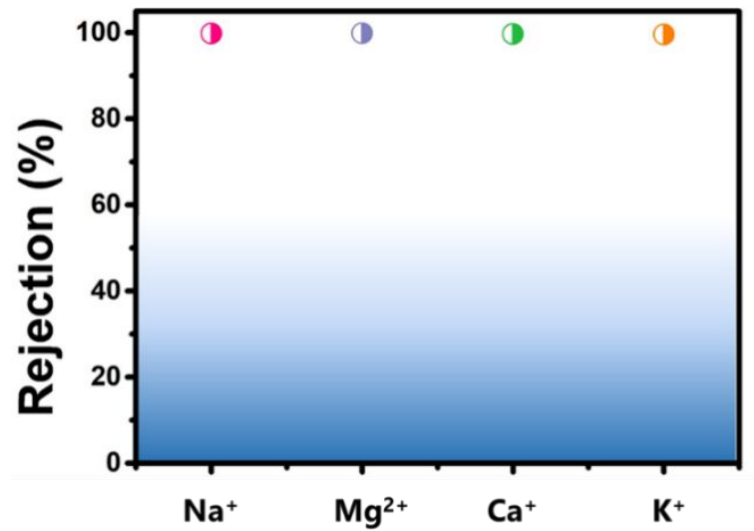

Figure S23. The ion rejection of actual seawater undergoing the solar energy-driven wastewater purification under one sun illumination.

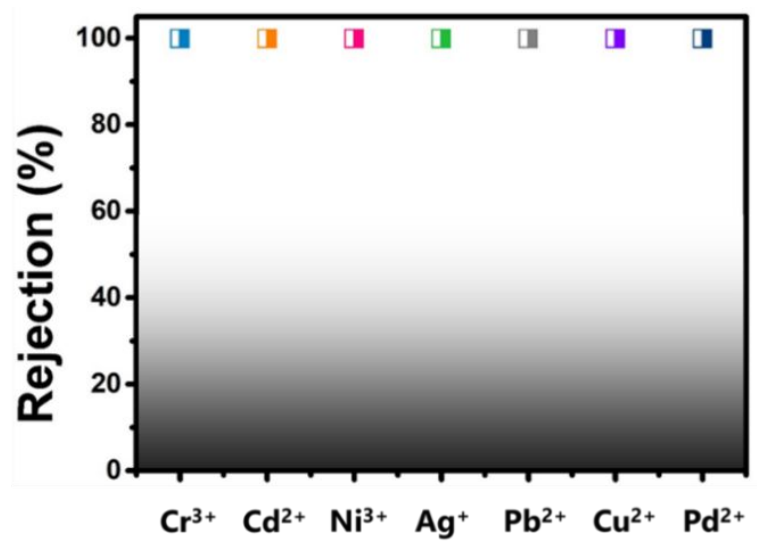

Figure S24. The ion rejection of artificial heavy metal ion solution undergoing the solar energydriven wastewater purification under one sun illumination. 


\begin{tabular}{|c|c|c|}
\hline Sample & Tensile strain at break & Tensile strength \\
\hline CC-BPHPLS & $167.5 \%$ & $6.22 \mathrm{MPa}$ \\
\hline
\end{tabular}

Table S1. The characterization of mechanical properties of CC-BPHPLS.

\begin{tabular}{|c|c|c|c|}
\hline \multicolumn{2}{|r|}{ Materials } & $\begin{array}{c}\text { Evaporation } \\
\text { rate } \\
\left(\mathrm{Kg} \mathrm{m}^{-2} \mathrm{~h}^{-1}\right) \\
\end{array}$ & Ref. \\
\hline \multirow{5}{*}{ Semiconductors } & Black TiOx nanoparticles & 0.8 & 1 \\
\hline & Black titania film & 1.13 & 2 \\
\hline & $\begin{array}{c}\text { Narrow-bandgap } \mathrm{Ti}_{2} \mathrm{O}_{3} \\
\text { nanoparticles }\end{array}$ & 1.32 & 3 \\
\hline & $\begin{array}{l}\text { Black amorphous Al-Ti-O } \\
\text { nanostructure }\end{array}$ & 1.03 & 4 \\
\hline & MoOx HNS Membrane & 1.255 & 5 \\
\hline \multirow{5}{*}{$\begin{array}{c}\text { Metallic } \\
\text { plasmonic }\end{array}$} & Au nanoflowers silica gel & 1.356 & 6 \\
\hline & Black silver nanostructures & 1.32 & 7 \\
\hline & Thin-film black gold membrane & 0.67 & 8 \\
\hline & $\mathrm{Au} / \mathrm{AAO}$ & 0.63 & 9 \\
\hline & Au/filter film & 1.15 & 10 \\
\hline \multirow{5}{*}{ Polymers } & PDAVBNC membrane & 1 & 11 \\
\hline & PPY coated SS mesh & 0.92 & 12 \\
\hline & Kapok fibers-PPy aerogels & 1.3752 & 13 \\
\hline & Monolithic polymer foam & 1.17 & 14 \\
\hline & PPy-coated VMP foam & 1.41 & 15 \\
\hline \multirow{7}{*}{ Carbon-based } & \begin{tabular}{|c|} 
Bilayer wood with a carbonized \\
surface
\end{tabular} & 1 & 16 \\
\hline & $\begin{array}{l}\text { rGO-MWCNT PVDF } \\
\text { membranes }\end{array}$ & 1.22 & 17 \\
\hline & CB/PMMA-PAN membrane & 1.3 & 18 \\
\hline & Functionalized rGO & 0.47 & 19 \\
\hline & PFS@rGO & 1.375 & 20 \\
\hline & wood/CNTs membrane & 0.95 & 21 \\
\hline & This work & 1.484 & - \\
\hline
\end{tabular}

Table S2. The comparison of the solar-thermal conversion efficiency using various photothermal materials under one sun illumination.

\begin{tabular}{|c|c|c|}
\hline Hybrid system & Power density $(\mathrm{W} \mathrm{m-2)}$ & Evaporation efficiency(\%) \\
\hline$[22]$ & 0.5 & 75 \\
\hline$[23]$ & 0.4 & 84.1 \\
\hline$[24]$ & 0.00024 & 85 \\
\hline$[25]$ & 0.4 & 87.4 \\
\hline This work & 0.12 & 93 \\
\hline
\end{tabular}

Table S3. Comparison of power output of simultaneous steam-electricity-generation strategies.

\section{References:}

[1] Ye, M. M.; Jia, J.; Wu, Z. J.; Qian, C. X.; Chen, R.; O'Brien, P. G.; Sun, W.; Dong, Y. C.; Ozin, 
G. A. Synthesis of Black $\mathrm{TiO}_{\mathrm{x}}$ Nanoparticles by Mg Reduction of $\mathrm{TiO}_{2}$ Nanocrystals and their Application for Solar Water Evaporation. Advanced Energy Materials 2017, 7, 1601811. http://doi.org/10.1002/aenm.201601811

[2] Zhu, G. L.; Xu, J. J.; Zhao, W. L.; Huang, F. Q. Constructing Black Titania with Unique Nanocage Structure for Solar Desalination. ACS Appl Mater Interfaces 2016, 8, 31716-31721. http://doi.org/10.1021/acsami.6b11466

[3] Wang, J.; Li, Y. Y.; Deng, L.; Wei, N. N.; Weng, Y. K.; Dong, S.; Qi, D. P.; Qiu, J.; Chen, X. D.; $\mathrm{Wu}, \mathrm{T}$. High-Performance Photothermal Conversion of Narrow-Bandgap $\mathrm{Ti}_{2} \mathrm{O}_{3}$ Nanoparticles. Adv Mater 2017, 29, 1603730. http://doi.org/10.1002/adma.201603730

[4] Yi, L. C.; Ci, S. Q.; Luo, S. L.; Shao, P.; Hou, Y.; Wen, Z. H. Scalable and Low-cost Synthesis of Black Amorphous Al-Ti-O Nanostructure for High-efficient Photothermal Desalination. Nano Energy 2017, 41, 600-608. http://doi.org/10.1016/j.nanoen.2017.09.042

[5] Lu, Q. C.; Yang, Y.; Feng, J. R.; Wang, X. Oxygen-Defected Molybdenum Oxides Hierarchical Nanostructure Constructed by Atomic-Level Thickness Nanosheets as an Efficient Absorber for Solar Steam Generation. Solar RRL 2019, 3, 1800277. http://doi.org/10.1002/solr.201800277

[6] Gao, M. M.; Peh, C. K.; Phan, H. T.; Zhu, L. L.; Ho, G. W. Solar Absorber Gel: Localized Macro-Nano Heat Channeling for Efficient Plasmonic Au Nanoflowers Photothermic Vaporization and Triboelectric Generation. Advanced Energy Materials 2018, 8, 1800711. http://doi.org/10.1002/aenm.201800711

[7] Chen, J. X.; Feng, J.; Li, Z. W.; Xu, P. P.; Wang, X. J.; Yin, W. W.; Wang, M. Z.; Ge, X. W.; Yin, Y. D. Space-Confined Seeded Growth of Black Silver Nanostructures for Solar Steam Generation. Nano Lett 2019, 19, 400-407. http://doi.org/10.1021/acs.nanolett.8b04157

[8] Bae, K.; Kang, G. M.; Cho, S. K.; Park, W.; Kim, K.; Padilla, W. J. Flexible Thin-film Black Gold Membranes with Ultrabroadband Plasmonic Nanofocusing for Efficient Solar Vapour Generation. Nature Communications, 2015, 6, 10103. http://doi.org/10.1038/ncomms10103

[9] Zhou, L.; Zhuang, S. D.; He, C.; Tan, Y. L.; Wang, Z. L.; Zhu, J. Self-assembled Spectrum Selective Plasmonic Absorbers with Tunable Bandwidth for Solar Energy Conversion. Nano Energy 2017, 32, 195-200. http://doi.org/ 10.1016/j.nanoen.2016.12.031

[10] Liu, C. X.; Huang, J. F.; Hsiung, C.-E.; Tian, Y.; Wang, J. J.; Han, Y.; Fratalocchi, A. High- 
Performance Large-Scale Solar Steam Generation with Nanolayers of Reusable Biomimetic $\begin{array}{llllll}\text { Nanoparticles. Advanced Sustainable Systems } & \text { 2017, } & 1600013 .\end{array}$ http://doi.org/10.1002/adsu.201600013

[11] Wu, X. H.; Cao, S. S.; Ghim, D.; Jiang, Q. S.; Singamaneni, S.; Jun, Y.-S. A Thermally Engineered Polydopamine and Bacterial Nanocellulose Bilayer Membrane for Photothermal Membrane Distillation with Bactericidal Capability. Nano Energy 2021, 79, 105353. http://doi.org/10.1016/j.nanoen.2020.105353

[12] Zhang, L. B.; Tang, B.; Wu, J. B.; Li, R. Y.; Wang, P. Hydrophobic Light-to-Heat Conversion Membranes with Self-Healing Ability for Interfacial Solar Heating. Adv Mater 2015, 27, 4889-94. http://doi.org/10.1002/adma.201502362

[13] Mu, P.; Bai, W.; Fan, Y. K.; Zhang, Z.; Sun, H. X.; Zhu, Z. Q.; Liang, W. D.; Li, A. Conductive Hollow Kapok Fiber-PPy Monolithic Aerogels with Excellent Mechanical Robustness for Efficient Solar Steam Generation. Journal of Materials Chemistry A 2019, 7, 9673-9679. http://doi.org/10.1039/c8ta12243a

[14] Chen, Q. M.; Pei, Z. Q.; Xu, Y. S.; Li, Z.; Y, Y.; W, Y.; Ji, Y.; A Durable Monolithic Polymer Foam for Efficient Solar Steam Generation. Chemical Science, 2018, 9, 623-628. http://doi.org/10.1039/c7sc02967e

[15] He, J. X.; Zhang, Z.; Xiao, C. H.; Liu, F.; Sun, H. X.; Zhu, Z. Q.; Liang, W. D.; Li, A. A HighPerformance Salt-Rejecting and Cost-Effective Superhydrophilic Porous Monolithic Polymer Foam for Solar Steam Generation. ACS Appl Mater Interfaces 2020, 12, 16308-16318. http://doi.org/10.1021/acsami.9b22832

[16] Li, X. Q.; Lin, R. X.; Ni, G; Xu, N.; Hu, X. Z.; Zhu, B.; Lv, G. X.; Li, J. L.; Zhu, S. N.; Zhu, J.; Three-dimensional Artificial Transpiration for Efficient Solar Waste Water Treament. National Science Review 2018, 5, 70-77. https://doi.org/10.1093/nsr/nwx051

[17] Wang, Y. C.; Wang, C. Z.; Song, X. J.; Megarajan, S. K.; Jiang, H. Q. A Facile Nanocomposite Strategy to Fabricate a rGO-MWCNT Photothermal Layer for Efficient Water Evaporation. Journal of Materials Chemistry A 2018, 6, 963-971.http://doi.org/10.1039/c7ta08972d

[18] Xu, W. C.; Hu, X. Z.; Zhuang, S. D.; Wang, Y. X.; Li, X. Q.; Zhou, L.; Zhu, S. N.; Zhu, J. Flexible and Salt Resistant Janus Absorbers by Electrospinning for Stable and Efficient Solar 
Desalination. Advanced Energy Materials 2018, 8, 1702884. http://doi.org/ 10.1002/aenm.201702884

[19] Yang, J. L.; Pang, Y. S.; Huang, W. X.; Shaw, S. K.; Schiffbauer, J.; Pillers, M. A.; Mu, X.; Luo, S. R.; Zhang, T.; Huang, Y. J.; Li, G. X.; Ptasinska, S.; Lieberman, M.; Luo, T. F. Functionalized Graphene Enables Highly Efficient Solar Thermal Steam Generation. ACS Nano 2017, 11, 5510-5518. https://doi.org/10.1021/acsnano.7b00367

[20] Chen, T. J.; Wang, S.; Wu, Z. Z.; Wang, X. D.; Peng, J.; Wu, B. H.; Cui, J. Q.; Fang, X. L.; Xie, Y. Q.; Zheng, N. F. A Cake Making Strategy to Prepare Reduced Graphene Oxide Wrapped Plant Fiber Sponges for High-efficiency Solar Steam Generation. Journal of Materials Chemistry A 2018, 6, 14571-14576. http://doi.org/10.1039/c8ta04420a

[21] Chen, C. J; Li, Y. J; Song, J. W; Yang, Z.; Kuang, Y. D.; Hitz, E.; Jia, C.; Gong, A.; Jiang, F.; Zhu, J. Y.; Yang, B.; Xie, J.; Hu, L. B. Highly Flexible and Efficient Solar Steam Generation Device. Adv Mater 2017, 29, 1701756. http://doi.org/ 10.1002/adma.201701756

[22] Yang, P. H.; Liu, K.; Chen, Q.; Li, J.; Duan, J. J.; Xue, G. B.; Xu, Z. S.; Xie, W. K.; Zhou, J. Solar-driven Simultaneous Steam Production and Electricity Generation from Salinity. Energy \& Environmental Science 2017, 10, 1923-1927. https://doi.org/10.1039/C7EE01804E

[23] Zhang, Y.; Ravi S. K.; Tan S. C. Food-derived Carbonaceous Materials for Solar Desalination and Thermo-electric Power Generation. Nano Energy 2019, 65, 104006. https://doi.org/10.1016/j.nanoen.2019.104006

[24] Gao, M. M.; Peh, C. K. N.; Phan, H. T.; Zhu, L. L.; Ho, G. W. Solar Absorber Gel: Localized macro-nano Heat Channeling for Efficient Plasmonic Au Nanoflowers Photothermic Vaporization and Triboelectric Generation. Advanced Energy Materials 2018, 8, 1800711.1-1800711.9.

[25] Zhu, L. L.; Ding, T. P.; Gao, M. M.; Peh, C. K. N.; Ho, G. W. Shape Conformal and Thermal Insulative Organic Solar Absorber Sponge for Photothermal Water Evaporation and Thermoelectric Power Generation. Advanced Energy Materials 2019, 9, 1900250. https://doi.org/10.1002/aenm.201900250 